\title{
Lie group analysis for MHD effects on the convectively heated stretching porous surface with the heat source/sink
}

\author{
Limei $\mathrm{CaO}^{1}$, Xinhui $\mathrm{Si}^{1 *}$, Liancun Zheng ${ }^{1}$ and Huihui Pang ${ }^{2}$
}

\section{"Correspondence:}

sixinhui_ustb@126.com

'School of Mathematics and

Physics, University of Science and

Technology Beijing, Beijing, 100083,

China

Full list of author information is

available at the end of the article

\begin{abstract}
This paper investigates magnetohydrodynamic (MHD) boundary layer flow and convective heat transfer of a fluid with variable viscosity through a porous medium towards a stretching sheet by considering the effects of viscous dissipation in presence of heat source/sink. The fluid viscosity is assumed to be a linear function of the temperature. The application of scaling group of transformations on the generalized stretching surface with injection velocity leads to two possible surface conditions. The governing equations with two types of boundary conditions are solved numerically using Bvp4c with MATLAB, respectively. Furthermore, more attention is paid to the effects of some physical parameters on the velocity and the temperature distribution with considering the permeability and the heat sink or the heat source.
\end{abstract}

Keywords: Lie group analysis; stretching porous surface; convective heat transfer; heat source/sink

\section{Introduction}

The interest in MHD fluid flows stems from the fact that liquid metals that occur in nature and industry are electrically conducting, which is attractive both from a mathematical and a physical standpoint. This type of flow has received much attention of many researchers due to its applications in technological and engineering problems such as MHD generators, plasma studies, nuclear reactors, geothermal energy extractions. By the application of a magnetic field, hydromagnetic techniques are used for the purification of molten metals from non-metallic inclusions. Then the type of problem that we are dealing with is very useful to polymer technology and metallurgy [1-4]. In addition, some theoretical work also has been done. For example, Rasmussen [5] numerically studied the problem of the steady viscous symmetric flow between two parallel porous coaxial disks. Hayat et al. [6] studied the MHD flow of an upper-convected Maxwell fluid over a porous stretching plate with the homotopy analysis method. Noor [7] presented an analysis of the MHD flow of a Maxwell fluid past a vertical stretching sheet in the presence of thermophoresis and chemical reactions. Hayat et al. [8] constructed an analytic solution for unsteady MHD flow in a rotating Maxwell fluid through a porous medium. Ibrahim and Shankar [9] investigated MHD boundary layer flow and heat transfer of a nanofluid past a permeable stretching sheet with slip boundary conditions.

(C) 2015 Cao et al.; licensee Springer. This article is distributed under the terms of the Creative Commons Attribution 4.0 International License (http://creativecommons.org/licenses/by/4.0/), which permits unrestricted use, distribution, and reproduction in any medium, provided you give appropriate credit to the original author(s) and the source, provide a link to the Creative Commons license, and indicate if changes were made. 
Lie group analysis, also called symmetry analysis, was developed by Sophus Lie to find point transformations which map a given differential equation to itself. This method has been used by many researchers to solve some nonlinear problems in fluid mechanics [10-17]. Furthermore, the scaling group techniques, a special form of Lie group transformations, have been applied by many researchers [18-26] to study different flow phenomena over different nonlinear dynamical systems, aerodynamics, and some other engineering branches. For example, Kanadasamy and Muhaimin [21] discussed steady twodimensional flow of incompressible fluid over a vertical stretching sheet by scaling group of transformations. Hamad [23] used this method to study the effect of a magnetic field on the free convection flow of a nanofluid over a linear stretching. Das [24] analyzed the MHD boundary layer flow of an electrically conducting nanofluid past a vertical convectively heated permeable stretching surface with variable stream conditions in presence of chemical reaction.

Motivated by the above works, the aim of this paper is to investigate the MHD effects on the convectively heated stretching porous surface with the heat source/sink. With the assistance of scaling group of transformations, two types of boundary conditions satisfying the similarity transformations are obtained, and then the coupled differential equations are deduced according to different boundary conditions, respectively. The effects of different parameters on the velocity and temperature distribution for these two cases are plotted in graphs and discussed in detail.

\section{Preliminaries}

Consider a steady two-dimensional forced convection flow of a viscous incompressible laminar dissipating fluid past a convectively heated stretching sheet immersed in a porous medium in the region $y>0$. Keeping the origin fixed, a force is applied along the $x$-axis which results stretching of the sheet. All body forces except by magnetic field are neglected etc. and the uniform magnetic field of strength $B_{0}$ is assumed to be perpendicular to the $x$-axis. We assume the temperature of the sheet to be different from that of the ambient medium and the fluid viscosity to vary with temperature, while the other fluid properties are assumed to be constants. $u$ and $v$ are the components of velocity in $x$ and $y$ directions and $T$ is the temperature of the fluid, respectively.

The continuity, momentum, and energy governing equations are written as [27]

$$
\begin{aligned}
& \frac{\partial u}{\partial x}+\frac{\partial v}{\partial y}=0 \\
& u \frac{\partial u}{\partial x}+v \frac{\partial u}{\partial y}=\frac{1}{\rho} \frac{\partial}{\partial y}\left(\mu \frac{\partial u}{\partial y}\right)-\frac{\sigma B_{0}^{2}}{\rho} u-\frac{\mu}{\rho k} u, \\
& u \frac{\partial T}{\partial x}+v \frac{\partial T}{\partial y}=\frac{\kappa}{\rho c_{p}} \frac{\partial^{2} T}{\partial y^{2}}+\frac{\mu}{\rho c_{p}}\left(\frac{\partial u}{\partial y}\right)^{2}+\frac{Q_{0}}{\rho c_{p}}\left(T-T_{\infty}\right),
\end{aligned}
$$

where $\kappa$ is the effective thermal conductivity of the fluid, $Q_{0}$ is the dimensional heat generation $\left(Q_{0}>0\right)$ or absorption $\left(Q_{0}<0\right)$ coefficient, $c_{p}$ is the specific heat, $\rho$ is the fluid density, $\mu$ is the coefficient of fluid viscosity (dependent on temperature), and $k$ is the permeability of the porous medium. 
The boundary conditions can be written as

$$
\begin{aligned}
& u=U(x), \quad v=V(x), \quad-\kappa \frac{\partial T}{\partial y}=h_{f}\left(T_{w}-T\right) \quad \text { at } y=0, \\
& u \rightarrow 0, \quad T \rightarrow T_{\infty} \quad \text { as } y \rightarrow \infty .
\end{aligned}
$$

We assume the bottom surface of the plate is heated by convection from a hot fluid with temperature $T_{w}$, which provides a heat transfer coefficient $h_{f}$.

The temperature dependent fluid viscosity is given by Batchelor [28]

$$
\mu=\mu^{*}\left[a+b\left(T_{w}-T\right)\right]
$$

where $\mu^{*}$ is the constant value of the coefficient of viscosity far away from the sheet and $a, b>0$ are constants.

Therefore, (2.2) becomes

$$
u \frac{\partial u}{\partial x}+v \frac{\partial u}{\partial y}=\frac{1}{\rho} \frac{\partial \mu}{\partial T} \frac{\partial T}{\partial y} \frac{\partial u}{\partial y}+\frac{\mu}{\rho} \frac{\partial^{2} u}{\partial y^{2}}-\frac{\sigma B_{0}^{2}}{\rho} u-\frac{\mu}{\rho k} u .
$$

We introduce the streamwise function $\Psi$ and the dimensionless temperature $\Theta$ as follows:

$$
u=\frac{\partial \Psi}{\partial y}, \quad v=-\frac{\partial \Psi}{\partial x}, \quad \Theta=\frac{T-T_{\infty}}{T_{w}-T_{\infty}} .
$$

Substituting relations (2.7) into (2.2)-(2.3), we obtain

$$
\begin{aligned}
& \frac{\partial \Psi}{\partial y} \frac{\partial^{2} \Psi}{\partial x \partial y}-\frac{\partial \Psi}{\partial x} \frac{\partial^{2} \Psi}{\partial y^{2}} \\
& \quad=-A v^{*} \frac{\partial \Theta}{\partial y} \frac{\partial^{2} \Psi}{\partial y^{2}}+v^{*}[a+A(1-\Theta)] \frac{\partial^{3} \Psi}{\partial y^{3}}-\frac{\nu^{*}}{k}[a+A(1-\Theta)] \frac{\partial \Psi}{\partial y}-\frac{\sigma B_{0}^{2}}{\rho} \frac{\partial \Psi}{\partial y} \\
& \frac{\partial \Psi}{\partial y} \frac{\partial \Theta}{\partial x}-\frac{\partial \Psi}{\partial x} \frac{\partial \Theta}{\partial y} \\
& \quad=\frac{\kappa}{\rho c_{p}} \frac{\partial^{2} \Theta}{\partial y^{2}}+\frac{Q_{0}}{\rho c_{p}} \Theta+\frac{v^{*}}{c_{p}\left(T_{w}-T_{\infty}\right)}[a+A(1-\Theta)]\left(\frac{\partial^{2} \Psi}{\partial y^{2}}\right)^{2}
\end{aligned}
$$

where $A=b\left(T_{w}-T_{\infty}\right), v^{*}=\frac{\mu^{*}}{\rho}$.

The boundary conditions become

$$
\begin{aligned}
& \frac{\partial \Psi}{\partial y}=U(x), \quad \frac{\partial \Psi}{\partial x}=-V(x), \quad-\kappa \frac{\partial \Theta}{\partial y}=h_{f}(1-\Theta) \quad \text { at } y=0, \\
& \frac{\partial \Psi}{\partial y} \rightarrow 0, \quad \Theta \rightarrow 0 \quad \text { as } y \rightarrow \infty .
\end{aligned}
$$

\section{Application of group transformations}

The scaling group of transformations presented by Mukhopadhyay et al. [20] is

$$
\begin{aligned}
& \Gamma: x^{*}=x e^{\epsilon \alpha_{1}}, \quad y^{*}=y e^{\epsilon \alpha_{2}}, \quad \Psi^{*}=\Psi e^{\epsilon \alpha_{3}}, \\
& u^{*}=u e^{\epsilon \alpha_{4}}, \quad v^{*}=v e^{\epsilon \alpha_{5}} \Theta^{*}=\Theta e^{\epsilon \alpha_{6}} .
\end{aligned}
$$


The above equation may be considered as a point transformation which transforms the coordinates $(x, y, \Psi, u, v, \Theta, \Phi)$ to $\left(x^{*}, y^{*}, \Psi^{*}, u^{*}, v^{*}, \Theta^{*}, \Phi^{*}\right)$.

Substituting (3.1) into (2.8) and (2.9), one can obtain

$$
\begin{aligned}
e^{\epsilon\left(\alpha_{1}+2 \alpha_{2}-2 \alpha_{3}\right)}\left(\frac{\partial \Psi^{*}}{\partial y^{*}} \frac{\partial^{2} \Psi^{*}}{\partial x^{*} \partial y^{*}}-\frac{\partial \Psi^{*}}{\partial x^{*}} \frac{\partial^{2} \Psi^{*}}{\partial y^{* 2}}\right) \\
=-A v^{*} e^{\epsilon\left(3 \alpha_{2}-\alpha_{3}-\alpha_{6}\right)} \frac{\partial \Theta^{*}}{\partial y^{*}} \frac{\partial^{2} \Psi^{*}}{\partial y^{* 2}} \\
+(a+A) v^{*} e^{\epsilon\left(3 \alpha_{2}-\alpha_{3}\right)} \frac{\partial^{3} \Psi^{*}}{\partial y^{* 3}}-A v^{*} e^{\epsilon\left(3 \alpha_{2}-\alpha_{3}-\alpha_{6}\right)} \Theta^{*} \frac{\partial^{3} \Psi^{*}}{\partial y^{* 3}} \\
\quad-\frac{v^{*}}{k}(a+A) e^{\epsilon\left(\alpha_{2}-\alpha_{3}\right)} \frac{\partial \Psi^{*}}{\partial y^{*}}+\frac{\nu^{*}}{k} A e^{\epsilon\left(\alpha_{2}-\alpha_{3}-\alpha_{6}\right)} \Theta^{*} \frac{\partial \Psi^{*}}{\partial y^{*}}-\frac{\sigma B_{0}^{2}}{\rho} e^{\epsilon\left(\alpha_{2}-\alpha_{3}\right)} \frac{\partial \Psi^{*}}{\partial y^{*}}, \\
e^{\epsilon\left(\alpha_{1}+\alpha_{2}-\alpha_{3}-\alpha_{6}\right)}\left(\frac{\partial \Psi^{*}}{\partial y^{*}} \frac{\partial \Theta^{*}}{\partial x^{*}}-\frac{\partial \Psi^{*}}{\partial x^{*}} \frac{\partial \Theta^{*}}{\partial y^{*}}\right) \\
\quad \frac{\kappa}{\rho c_{p}} e^{\epsilon\left(2 \alpha_{2}-\alpha_{6}\right)} \frac{\partial^{2} \Theta^{*}}{\partial y^{* 2}}+\frac{Q_{0}}{\rho c_{p}} e^{-\epsilon \alpha_{6}} \Theta^{*}+\frac{v^{*}}{c_{p}\left(T_{w}-T_{\infty}\right)}(a+A) e^{\epsilon\left(4 \alpha_{2}-2 \alpha_{3}\right)}\left(\frac{\partial^{2} \Psi^{*}}{\partial y^{* 2}}\right)^{2} \\
\quad-\frac{v^{*}}{c_{p}\left(T_{w}-T_{\infty}\right)} A e^{\epsilon\left(4 \alpha_{2}-2 \alpha_{3}-\alpha_{6}\right)} \Theta^{*}\left(\frac{\partial^{2} \Psi^{*}}{\partial y^{* 2}}\right)^{2},
\end{aligned}
$$

then the following relationship among the exponents can be obtained:

$$
\begin{aligned}
& \alpha_{1}+2 \alpha_{2}-2 \alpha_{3}=3 \alpha_{2}-\alpha_{3}-\alpha_{6}=3 \alpha_{2}-\alpha_{3}=\alpha_{2}-\alpha_{3}=\alpha_{2}-\alpha_{3}-\alpha_{6}, \\
& \alpha_{1}+\alpha_{2}-\alpha_{3}-\alpha_{6}=2 \alpha_{2}-\alpha_{6}=-\alpha_{6}=4 \alpha_{2}-2 \alpha_{3}=4 \alpha_{2}-2 \alpha_{3}-\alpha_{6} .
\end{aligned}
$$

Two results are presented subject to the two different boundary conditions in the following section.

\subsection{Case 1: equations with the first type of boundary conditions}

For case 1 , the stretching velocity and the suction/injection velocity are assumed to be

$$
U(x)=c x^{m}, \quad V(x)=-V_{0} x^{\frac{m-1}{2}}
$$

where $c>0$ and the power-law exponent $m$ both are constants. In this study we take $c=1$.

The corresponding boundary conditions (2.10) become

$$
\begin{aligned}
& \frac{\partial \Psi}{\partial y}=x^{m}, \quad \frac{\partial \Psi}{\partial x}=V_{0} x^{\frac{(m-1)}{2}}, \quad-\kappa \frac{\partial \Theta}{\partial y}=h_{f}(1-\Theta) \quad \text { at } y=0, \\
& \frac{\partial \Psi}{\partial y} \rightarrow 0, \quad \Theta \rightarrow 0 \quad \text { as } y \rightarrow \infty .
\end{aligned}
$$

The relation $3 \alpha_{2}-\alpha_{3}=\alpha_{2}-\alpha_{3}$ can result in $\alpha_{2}=0$. Hence, $\alpha_{1}+2 \alpha_{2}-2 \alpha_{3}=3 \alpha_{2}-\alpha_{3}-\alpha_{6}$ gives $\alpha_{6}=0, \alpha_{2}=\frac{1}{4} \alpha_{1}=\frac{1}{3} \alpha_{3}$. Also the boundary conditions yield $\alpha_{4}=m \alpha_{1}=\frac{1}{2} \alpha_{1}, \alpha_{5}=$ $\frac{m-1}{2} \alpha_{1}=-\frac{1}{4} \alpha_{1}$, and then $m=\frac{1}{2}$. 
Hence, the boundary conditions (3.6) are written as

$$
\begin{array}{ll}
\frac{\partial \Psi^{*}}{\partial y^{*}}=x^{* \frac{1}{2}}, & \frac{\partial \Psi^{*}}{\partial x^{*}}=V_{0} x^{*-\frac{1}{4}}, \quad-\kappa \frac{\partial \Theta^{*}}{\partial y^{*}}=h_{f}\left(1-\Theta^{*}\right) \quad \text { at } y^{*}=0, \\
\frac{\partial \Psi^{*}}{\partial y^{*}} \rightarrow 0, & \Theta^{*} \rightarrow 0 \quad \text { as } y^{*} \rightarrow \infty .
\end{array}
$$

Thus the set of transformations $\Gamma$ reduces to a one parameter group of transformations as follows:

$$
\begin{aligned}
& \Gamma: x^{*}=x e^{\epsilon \alpha_{1}}, \quad y^{*}=y e^{\epsilon \frac{\alpha_{1}}{4}}, \quad \Psi^{*}=\Psi e^{\epsilon \frac{3 \alpha_{1}}{4}}, \\
& u^{*}=u e^{\epsilon \frac{\alpha_{1}}{2}}, \quad v^{*}=v e^{-\epsilon \frac{\alpha_{1}}{4}}, \quad \Theta^{*}=\Theta .
\end{aligned}
$$

Expanding by Taylor's method in powers of $\epsilon$ and keeping terms up to $\epsilon$, one gets

$$
\begin{array}{ll}
x^{*}-x=x \epsilon \alpha_{1}, & y^{*}-y=y \epsilon \frac{\alpha_{1}}{4}, \\
u^{*}-u=u \epsilon \frac{\alpha_{1}}{2}, & v^{*}-v=-v \epsilon \frac{\alpha_{1}}{4}, \quad \Psi^{*}-\Psi=\Psi \epsilon \frac{3 \alpha_{1}}{4},
\end{array}
$$

The characteristic equations are

$$
\frac{\mathrm{d} x}{\alpha_{1} x}=\frac{\mathrm{d} y}{\frac{1}{4} \alpha_{1} y}=\frac{\mathrm{d} \Psi}{\frac{3}{4} \alpha_{1} \Psi}=\frac{\mathrm{d} u}{\frac{1}{2} \alpha_{1} u}=\frac{\mathrm{d} v}{-\frac{1}{4} \alpha_{1} v}=\frac{\mathrm{d} \Theta}{0} .
$$

Solving (3.10), we get

$$
y^{*} x^{*-\frac{1}{4}}=\eta^{*}, \quad \Psi^{*}=x^{*-\frac{3}{4}} F\left(\eta^{*}\right), \quad \Theta^{*}=\Theta\left(\eta^{*}\right),
$$

where $\eta^{*}$ is an invariable.

Substituting (3.11) into (3.2) and (3.3) yields

$$
\begin{aligned}
& (a+A) v^{*} F^{\prime \prime \prime}-A v^{*} \Theta F^{\prime \prime \prime}-A v^{*} \Theta^{\prime} F^{\prime \prime}+\frac{3}{4} F F^{\prime \prime}-\lambda(a+A) F^{\prime} \\
& -M^{2} F^{\prime}+\lambda A \Theta F^{\prime}-\frac{1}{2}\left(F^{\prime}\right)^{2}=0, \\
& E c(a+A) v^{*}\left(F^{\prime \prime}\right)^{2}-E c A v^{*} \Theta\left(F^{\prime \prime}\right)^{2}+\frac{3}{4} \Theta^{\prime} F+Q \Theta+\frac{\kappa}{\rho c_{p}} \Theta^{\prime \prime}=0,
\end{aligned}
$$

where $\lambda=\frac{v^{*} U}{k}$ is the permeability parameter, $M^{2}=\frac{\sigma B_{0}^{2} U}{\rho}$ is the Hartmann number, $E c=$ $\frac{U^{2}}{c_{p}\left(T_{w}-T_{\infty}\right)}$ is the Eckert number, and $Q=\frac{Q_{0} U}{\rho c_{p}}$ is the heat source/sink parameter.

The boundary conditions are transformed as

$$
\begin{aligned}
& F=\frac{4}{3} V_{0}, \quad F^{\prime}=1, \quad \Theta^{\prime}=\frac{h_{f}}{\kappa} x^{\frac{1}{4}}(\Theta-1) \quad \text { at } \eta^{*}=0, \\
& F^{\prime} \rightarrow 0, \quad \Theta \rightarrow 0, \quad \text { as } \eta^{*} \rightarrow \infty .
\end{aligned}
$$


In order to remove $v^{*}$ in (3.12) and (3.13), the following transformations for $\eta^{*}, F$, and $\Theta$ are introduced:

$$
\eta^{*}=v^{* \frac{1}{2}} \eta, \quad F=v^{* \frac{1}{2}} f, \quad \Theta=\theta,
$$

then (3.12) and (3.13) become

$$
\begin{aligned}
& (a+A) f^{\prime \prime \prime}-A \theta f^{\prime \prime \prime}-A \theta^{\prime} f^{\prime \prime}+\frac{3}{4} f^{\prime \prime}-\lambda(a+A) f^{\prime}-M^{2} f^{\prime}+\lambda A \theta f^{\prime}-\frac{1}{2}\left(f^{\prime}\right)^{2}=0 \\
& E c(a+A)\left(f^{\prime \prime}\right)^{2}-E c A \theta\left(f^{\prime \prime}\right)^{2}+\frac{3}{4} \theta^{\prime} f+Q \theta+\frac{1}{P r} \theta^{\prime \prime}=0
\end{aligned}
$$

where $\operatorname{Pr}=\frac{v^{*} \rho c_{p}}{\kappa}$ is the Prandtl number, and ' denotes the derivative with respect to $\eta$.

The boundary conditions are

$$
\begin{aligned}
& f=S, \quad f^{\prime}=1, \quad \theta^{\prime}=B(\theta-1) \quad \text { at } \eta=0, \\
& f^{\prime} \rightarrow 0, \quad \theta \rightarrow 0, \quad \text { as } \eta \rightarrow \infty,
\end{aligned}
$$

where $S=\frac{4}{3} V_{0} \nu^{*-\frac{1}{2}}$ is the suction/injection parameter and $B=\frac{h_{f}}{\kappa} \nu^{* \frac{1}{2}} x^{\frac{1}{4}}$ is the convection heat transfer parameter. Following the definition of Kundu et al. [25], we also assume $h_{f}=$ $c_{1} x^{-\frac{1}{4}}$ where $c_{1}$ is a constant.

\subsection{Case 2: equations with the second type of boundary conditions}

For case 2, we assume the streamwise velocity and the suction/injection velocity at the sheet are taken as

$$
U(x)=h x+C_{1} \frac{\partial u}{\partial y}+C_{2} \frac{\partial^{2} u}{\partial y^{2}}, \quad V(x)=-V_{0},
$$

where $h$ is a constant and $C_{1}, C_{2}$ are two independent coefficients named the slip coefficients.

Substituting the (2.7) into the boundary conditions (3.19) yields

$$
\begin{aligned}
& \frac{\partial \Psi}{\partial y}=h x+C_{1} \frac{\partial^{2} \Psi}{\partial y^{2}}+C_{2} \frac{\partial^{3} \Psi}{\partial y^{3}}, \quad \frac{\partial \Psi}{\partial x}=V_{0}, \\
& -\kappa \frac{\partial \Theta}{\partial y}=h_{f}(1-\Theta) \quad \text { at } y=0, \\
& \frac{\partial \Psi}{\partial y} \rightarrow 0, \quad \Theta \rightarrow 0 \quad \text { as } y \rightarrow \infty .
\end{aligned}
$$

From (3.4) and the boundary conditions (3.20), we get $\alpha_{1}=\alpha_{3}=\alpha_{4}$ and $\alpha_{2}=\alpha_{5}=\alpha_{6}=0$.

The characteristic equations are

$$
\frac{\mathrm{d} x}{\alpha_{1} x}=\frac{\mathrm{d} y}{0}=\frac{\mathrm{d} \Psi}{\alpha_{1} \Psi}=\frac{\mathrm{d} u}{\alpha_{1} u}=\frac{\mathrm{d} v}{0}=\frac{\mathrm{d} \Theta}{0} .
$$

Thus from (3.21) we obtain

$$
y^{*}=\eta^{*}, \quad \Psi^{*}=x F\left(\eta^{*}\right), \quad \Theta^{*}=\Theta\left(\eta^{*}\right),
$$

where $\eta^{*}$ still is an invariable. 


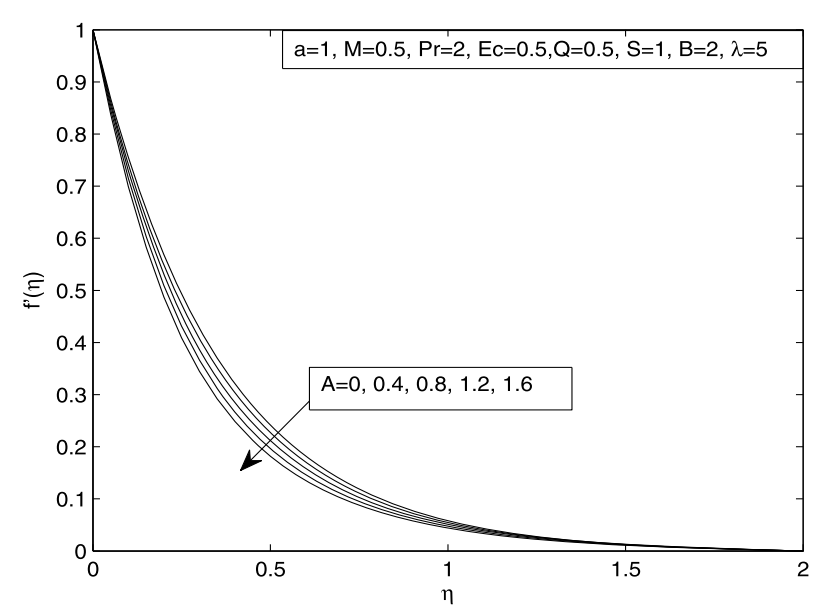

Figure 1 Variation of velocity $f^{\prime}(\eta)$ for different values of viscosity parameter $A$.

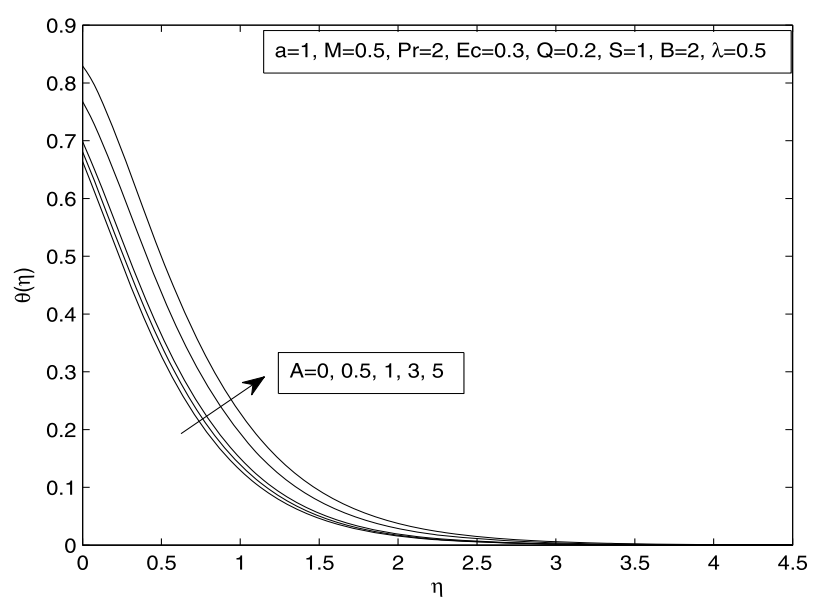

Figure 2 Variation of temperature $\theta(\eta)$ for different values of viscosity parameter $A$.

Using (3.22), (3.2) and (3.3) become

$$
\begin{aligned}
& (a+A) v^{*} F^{\prime \prime \prime}-A v^{*} \Theta F^{\prime \prime \prime}-A v^{*} \Theta^{\prime} F^{\prime \prime}+F F^{\prime \prime}-\frac{v^{*}}{k}(a+A) F^{\prime} \\
& -\frac{\sigma B_{0}^{2}}{\rho} F^{\prime}+\frac{v^{*}}{k} A \Theta F^{\prime}-\left(F^{\prime}\right)^{2}=0, \\
& \frac{x^{2}}{c_{p}\left(T_{w}-T_{\infty}\right)}(a+A) v^{*}\left(F^{\prime \prime}\right)^{2}-\frac{x^{2}}{c_{p}\left(T_{w}-T_{\infty}\right)} A v^{*} \Theta\left(F^{\prime \prime}\right)^{2} \\
& +\Theta^{\prime} F+\frac{Q_{0}}{\rho c_{p}} \Theta+\frac{\kappa}{\rho c_{p}} \Theta^{\prime \prime}=0 .
\end{aligned}
$$




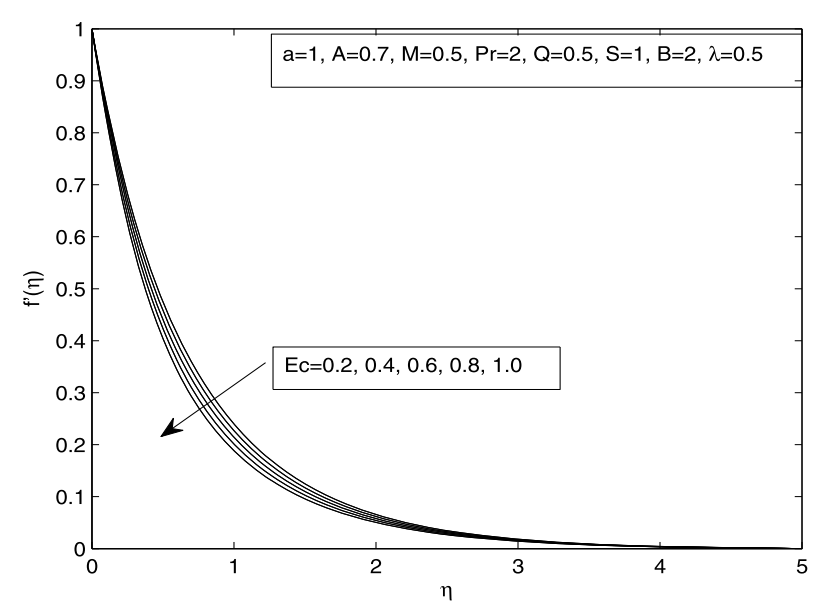

Figure 3 Variation of velocity $f^{\prime}(\eta)$ for different values of Eckert number Ec.

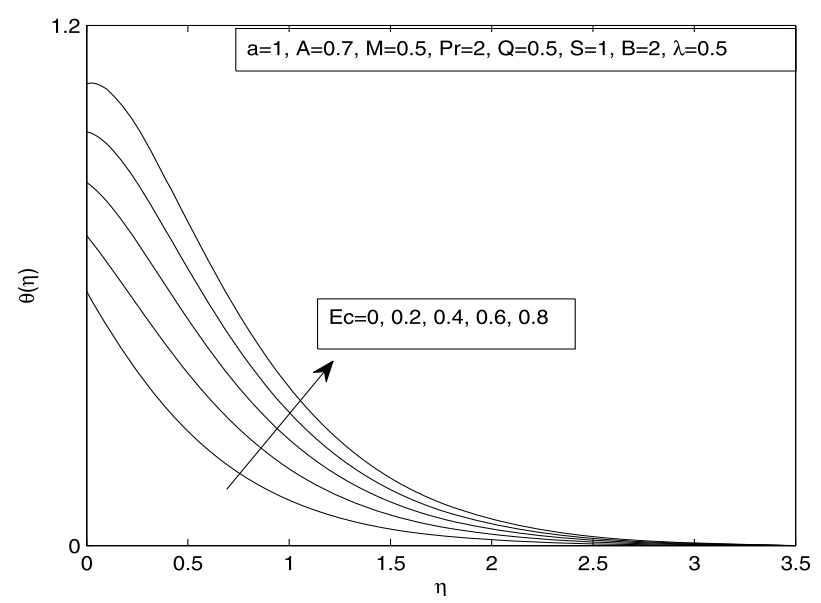

Figure 4 Variation of temperature $\theta(\eta)$ for different values of Eckert number Ec.

The boundary conditions are transformed as

$$
\begin{aligned}
& F=V_{0}, \quad F^{\prime}=h+C_{1} F^{\prime \prime}+C_{2} F^{\prime \prime \prime}, \quad \Theta^{\prime}=\frac{h_{f}}{\kappa}(\Theta-1) \quad \text { at } \eta^{*}=0, \\
& F^{\prime} \rightarrow 0, \quad \Theta \rightarrow 0, \quad \text { as } \eta^{*} \rightarrow \infty .
\end{aligned}
$$

Again, in order to remove $v^{*}$, the following transformations for $\eta^{*}, F$, and $\Theta$ in (3.12) and (3.13) are introduced:

$$
\eta^{*}=v^{* \frac{1}{2}} h^{-\frac{1}{2}} \eta, \quad F=v^{* \frac{1}{2}} h^{\frac{1}{2}} f, \quad \Theta=\theta .
$$

Equations (3.23) and (3.24) finally take the following form:

$$
\begin{gathered}
(a+A) f^{\prime \prime \prime}-A \theta f^{\prime \prime \prime}-A \theta^{\prime} f^{\prime \prime}+\not f^{\prime \prime}-\lambda(a+A) f^{\prime} \\
-M^{2} f^{\prime}+\lambda A \theta f^{\prime}-\left(f^{\prime}\right)^{2}=0,
\end{gathered}
$$




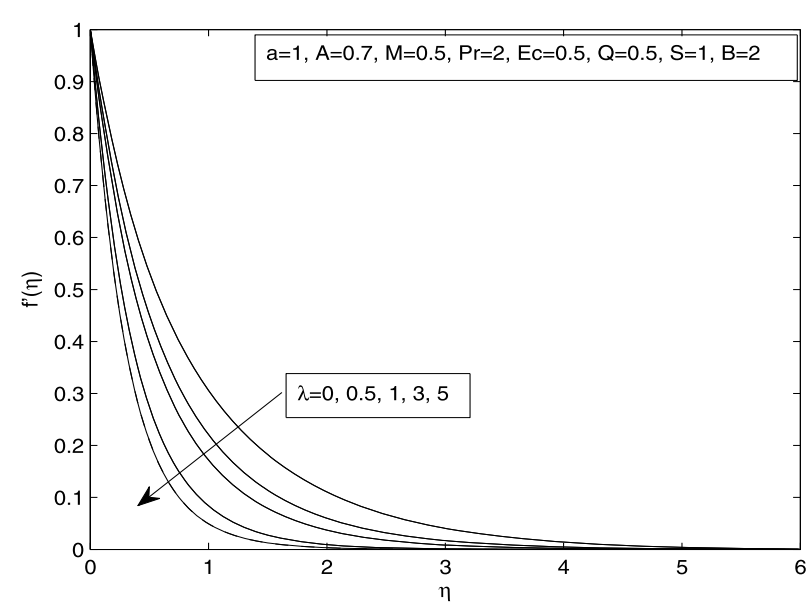

Figure 5 Variation of velocity $f^{\prime}(\eta)$ for different values of permeability parameter $\lambda$.

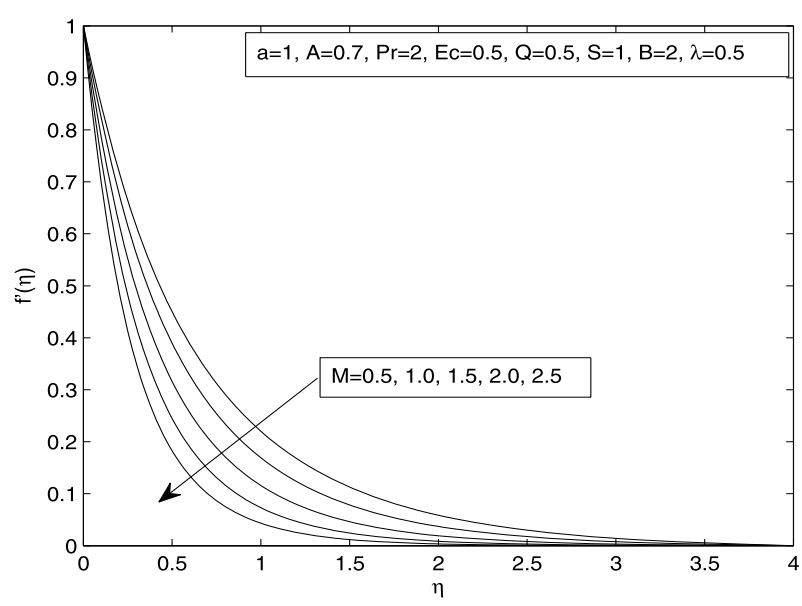

Figure 6 Variation of velocity $f^{\prime}(\eta)$ for different values of Hartmann number $M$.

$$
E c(a+A)\left(f^{\prime \prime}\right)^{2}-E c A \theta\left(f^{\prime \prime}\right)^{2}+\theta^{\prime} f+Q \theta+\frac{1}{P r} \theta^{\prime \prime}=0
$$

where ' denotes the derivative with respect to $\eta, \lambda=\frac{v^{*}}{k h}$ is the permeability parameter, $M^{2}=\frac{\sigma B_{0}^{2}}{\rho h}$ is the Hartmann number, $E c=\frac{h^{2} x^{2}}{c_{p}\left(T_{w}-T_{\infty}\right)}$ is the Eckert number, $Q=\frac{Q_{0}}{\rho c_{p} h}$ is the heat source/sink parameter, and $P r=\frac{v^{*} \rho c_{p}}{\kappa}$ is the Prandtl number.

The boundary conditions (3.25) become

$$
\begin{aligned}
& f=S, \quad f^{\prime}=1+k_{1} f^{\prime \prime}+k_{2} f^{\prime \prime \prime}, \quad \theta^{\prime}=B(\theta-1) \quad \text { at } \eta=0, \\
& f^{\prime} \rightarrow 0, \quad \theta \rightarrow 0, \quad \text { as } \eta \rightarrow \infty,
\end{aligned}
$$

where $S=v^{*-\frac{1}{2}} h^{-\frac{1}{2}} V_{0}$ is the suction/injection parameter, $k_{1}=C_{1} v^{*-\frac{1}{2}} h^{\frac{1}{2}}, k_{2}=C_{2} v^{*-1} h$ are the wall slip parameters, and $B=\frac{h_{f}}{\kappa} v^{* \frac{1}{2}} h^{-\frac{1}{2}}$ is the convection heat transfer parameter. 


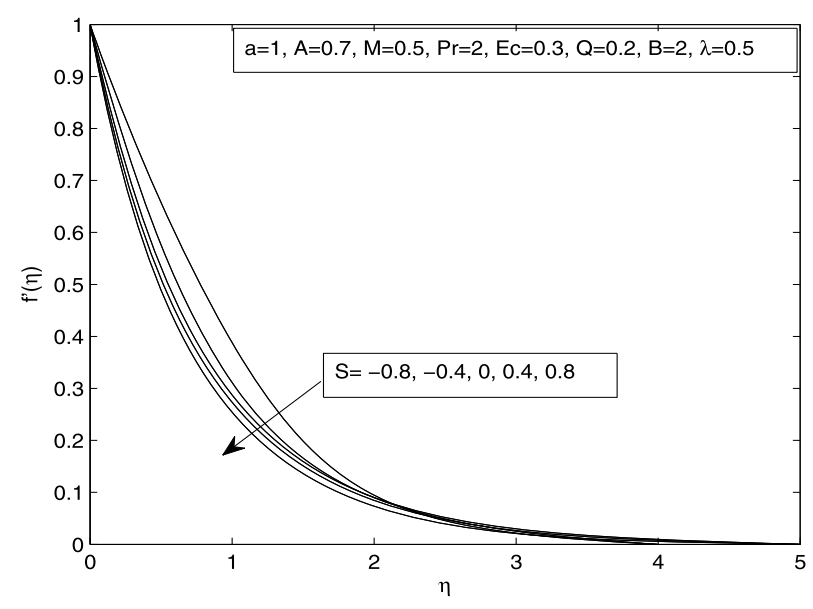

Figure 7 Variation of velocity $f^{\prime}(\eta)$ for different values of suction/injection parameter $S$.

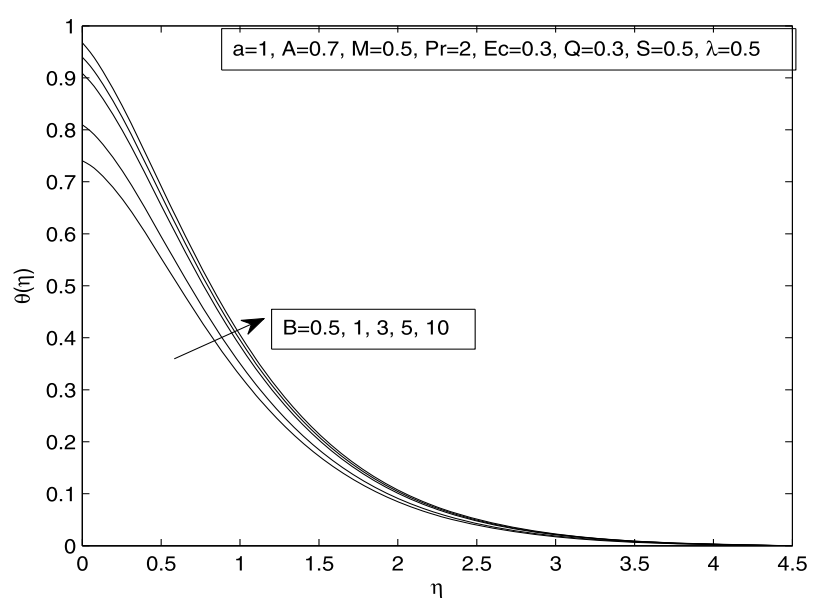

Figure 8 Variation of temperature $\theta(\eta)$ for different values of convection heat transfer parameter $B$.

\section{Numerical solutions and discussion}

Since (3.16)-(3.18) and (3.27)-(3.29) are coupled nonlinear boundary value problems, these equations are solved numerically by Bvp4c with MATLAB, which is a collocation method equivalent to the fourth order mono-implicit Runge-Kutta method. Since the velocity changes sharply in the boundary layer near the plate, this region with a sharp change makes this boundary value problem a relatively difficult one. In order to resolve better the boundary layer and obtain a more accurate solution, the relative error tolerance on the residuals is defined to be $10^{-6}$ (i.e. RelTol $=10^{-6}$ ) during the process of numerical computation. The results are presented graphically and in tables.

The effects of different physical parameters, such as the viscosity parameter, the permeability parameter, the Hartmann number and the Eckert number etc., on the velocity and temperature distribution are investigated for these two types of boundary conditions. To begin with, we study the case subject to the first type of boundary conditions. Figures 1 and 2 illustrate the effects of viscosity parameter $A$ on the velocity $f^{\prime}(\eta)$ and temperature distribution $\theta(\eta)$, respectively, as the other physical parameters are fixed. The viscosity 


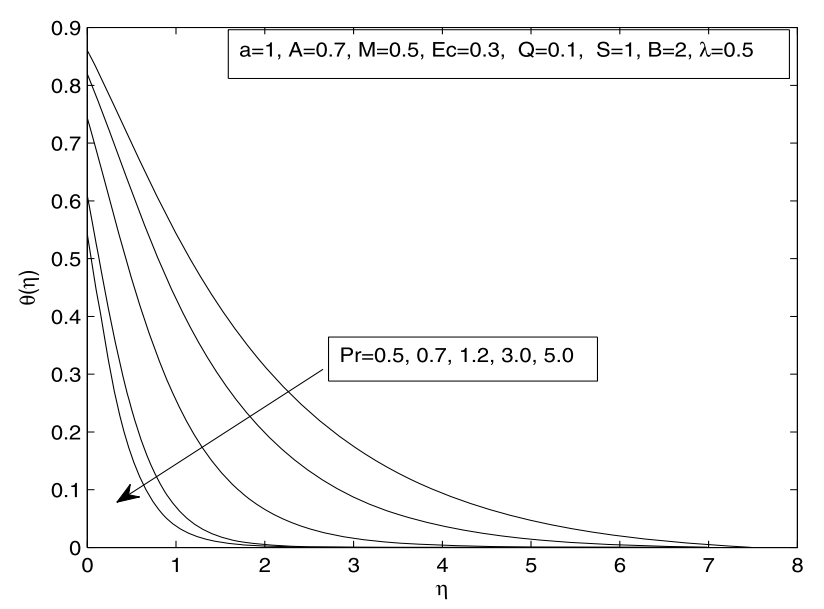

Figure 9 Variation of temperature $\theta(\eta)$ for different values of Prandtl number Pr.

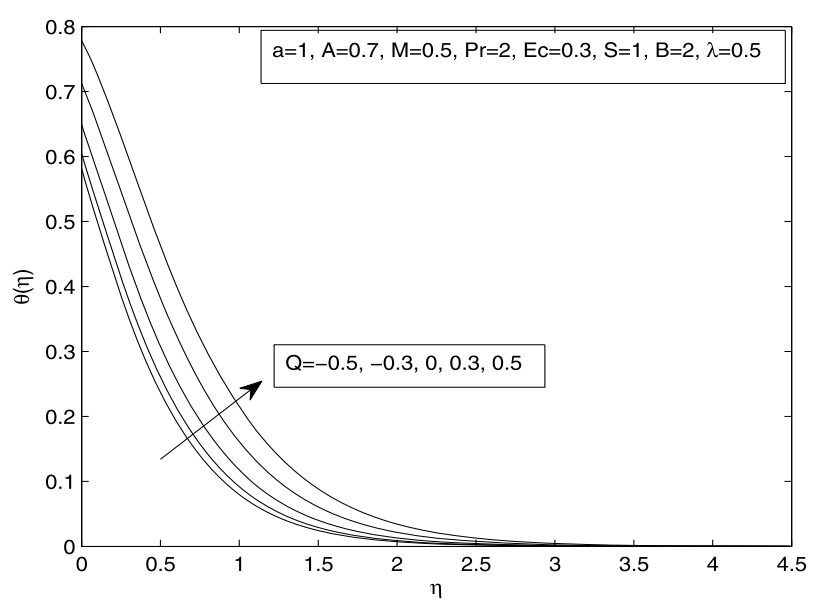

Figure 10 Variation of temperature $\theta(\eta)$ for different values of heat source/sink parameter $Q$.

Table 1 Comparison of $-f^{\prime \prime}(0)$ for $\operatorname{Pr}=10, L e=1$, and $N b=0.1$

\begin{tabular}{lllll}
\hline$\lambda$ & Present results & Dessie and Kishan [27] & Cortell [29] & Mukhopadhyay et al. [30] \\
\cline { 2 - 5 } & $\mathbf{- \mathbf { f } ^ { \prime \prime } ( \mathbf { 0 } )}$ & $\mathbf{- \mathbf { f } ^ { \prime \prime } ( \mathbf { 0 } )}$ & $\mathbf{- \mathbf { f } ^ { \prime \prime } ( \mathbf { 0 } )}$ & $\mathbf{- \mathbf { f } ^ { \prime \prime } ( \mathbf { 0 } )}$ \\
\hline 1 & 1.414214 & 1.414214 & 1.414213 & 1.414213 \\
2 & 1.732051 & 1.732051 & 1.732051 & 1.732051 \\
\hline
\end{tabular}

parameter $A$ is proportional to the difference of temperature. It is observed that there is little influence on the velocity distribution near the sheet. However, the influence becomes obvious as the velocity is far away from the sheet, which is an increasing function of the viscosity parameter. When we consider the effects of viscosity parameter on the temperature distribution, it becomes significant. The temperature is an increasing function of the viscosity parameter.

Figures 3 and 4 show the effects of the Eckert number on the velocity and temperature distribution. We can find that the velocity is the decreasing function and the temperature is an increasing function of the increasing Eckert number. In addition, at the sheet, the 
Table 2 Computed values of skin friction coefficient $-f^{\prime \prime}(0)$ for various values of $M, S$, and $k_{1}$

\begin{tabular}{lllll}
\hline $\mathbf{M}^{\mathbf{2}}$ & $\mathbf{S}$ & $\boldsymbol{k}_{\mathbf{1}}$ & Ibrahim and Shankar [9] & Present result \\
\hline 1 & 0 & 0 & 1.4142 & 1.414214 \\
1 & 0.2 & 0 & 1.5177 & 1.517745 \\
1 & 0.2 & 1 & 0.5656 & 0.565566 \\
1 & 0.2 & 1.2 & 0.5055 & 0.505457 \\
1.5 & 0.5 & 0 & 1.8508 & 1.850781 \\
2 & 0.5 & 0 & 2.0000 & 2.000000 \\
\hline
\end{tabular}

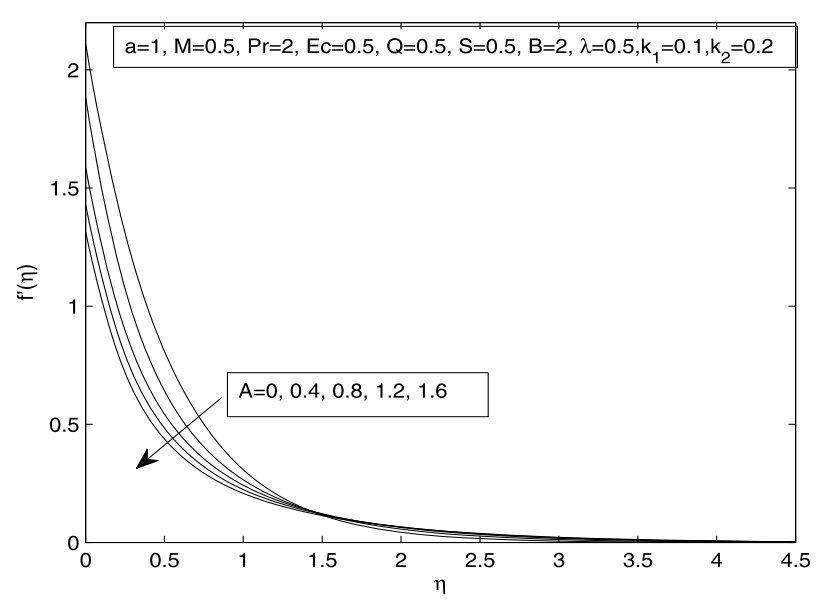

Figure 11 Variation of velocity $f^{\prime}(\eta)$ for different values of viscosity parameter $A$.

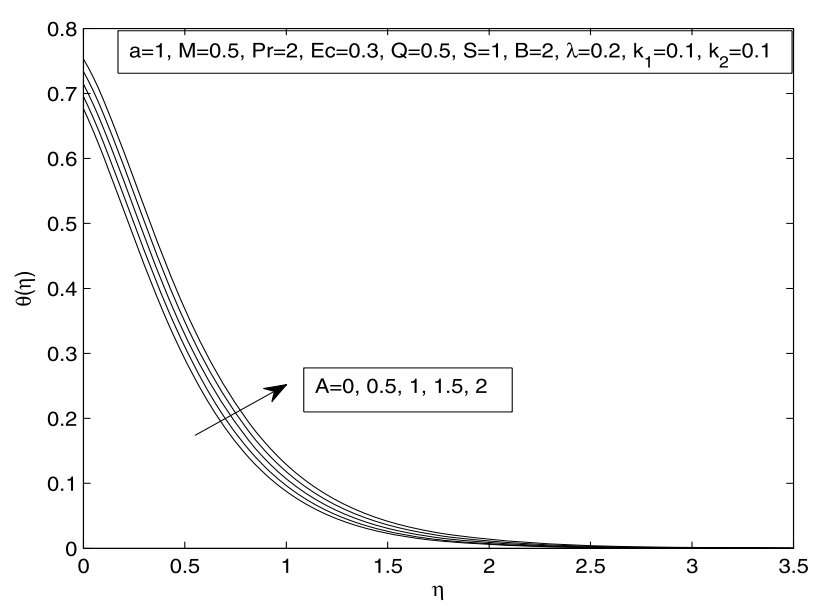

Figure 12 Variation of temperature $\theta(\eta)$ for different values of viscosity parameter $A$.

temperature also is different because of the existence of convection heat. As the variable $\eta$ trends from zero to infinity, the temperature becomes zero. This also reflects that the heat caused by friction can be neglected when the distance is far away from the sheet.

Figure 5 illustrates the effects of the permeability parameter on the velocity. The velocity decreases with the increasing value of the permeability parameter, which shows that the porous medium also has important effects on the velocity. Figure 6 shows the variation of velocity $f^{\prime}(\eta)$ for different values of the Hartmann number $M$. The velocity decreases 


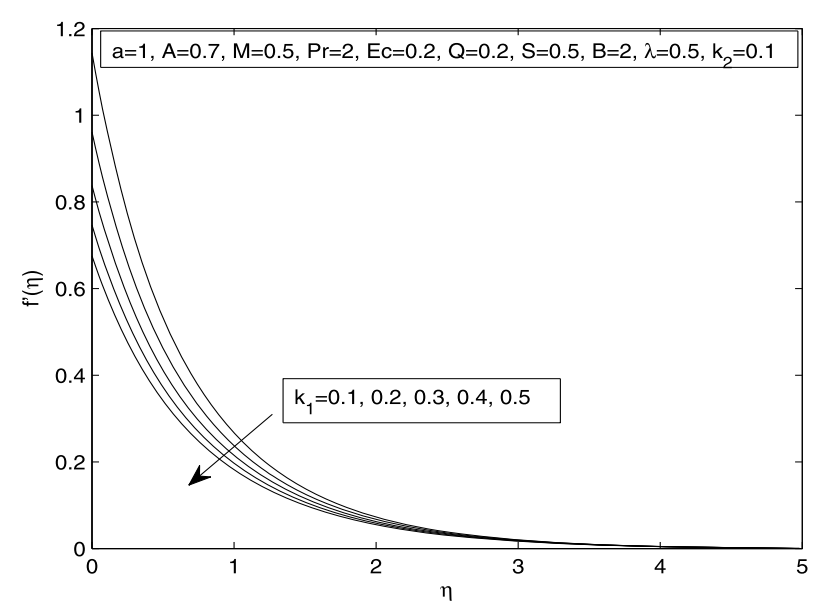

Figure 13 Variation of velocity $f^{\prime}(\eta)$ for different values of slip parameter $k_{1}$.

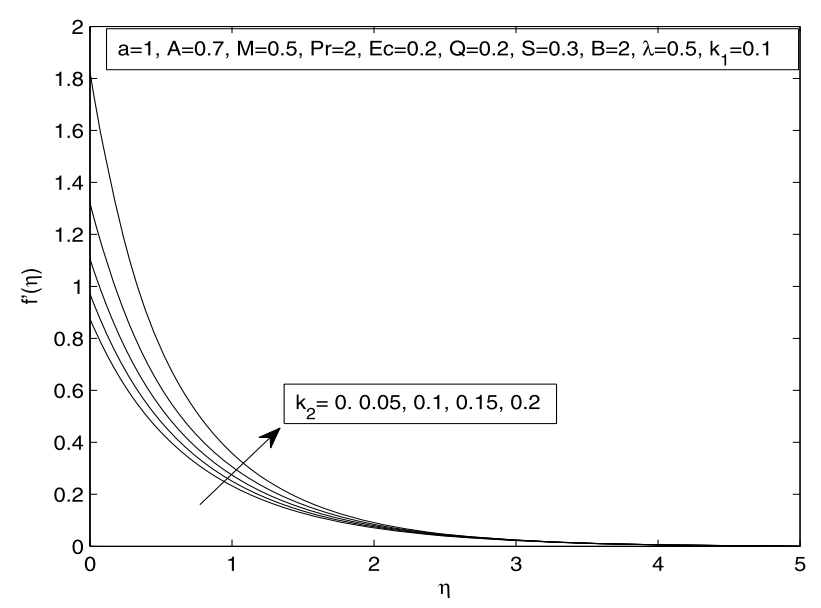

Figure 14 Variation of velocity $f^{\prime}(\eta)$ for different values of slip parameter $k_{2}$.

with the increase of $M$, which clearly indicates that the transverse magnetic field opposes the transport phenomena. The reason is that the variation of the Hartmann number leads to the variation of the Lorentz force due to magnetic field and the Lorentz force produces more resistance to transport phenomena. Since the suction velocity can restrain the increase of the velocity near the sheet, the velocity near the sheet is smaller than the case of injection velocity, which can be seen in Figure 7.

The convection heat transfer parameter $B$ also has important influence on the temperature distribution. The temperature is an increasing function of the parameter $B$, which is shown in Figure 8. The stronger convection results in a higher surface temperature, which causes the thermal effect to penetrate deeper into the quiescent fluid. Furthermore, since the convection heat transfer happens near the sheet, the influence on the temperature near the sheet is obvious, and when it is far away the sheet, the influence becomes smaller. Figure 9 exhibits the temperature distribution for variable values of Prandtl number. The temperature distribution is a decreasing function of the Prandtl number. Furthermore, the thermal boundary layer becomes thinner with the increasing Prandtl number, which also 


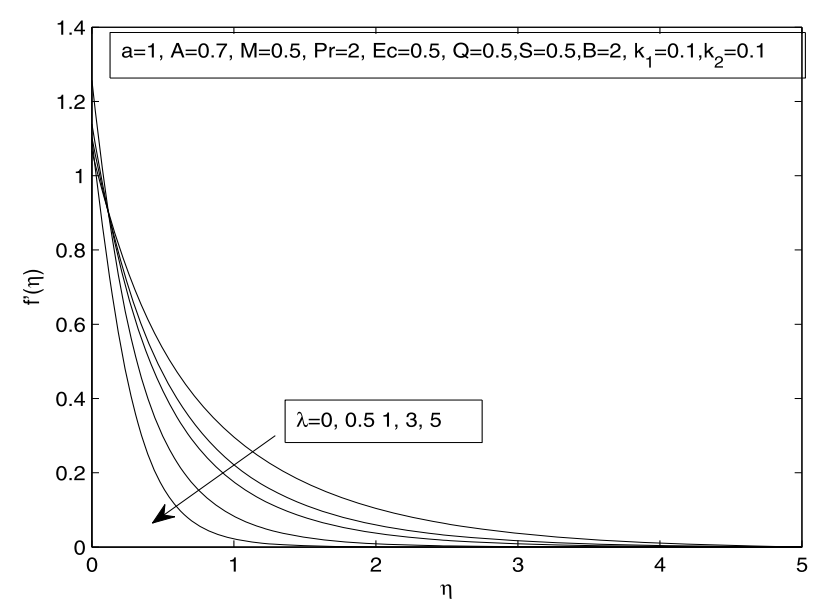

Figure 15 Variation of velocity $f^{\prime}(\eta)$ for different values of permeability parameter $\lambda$.

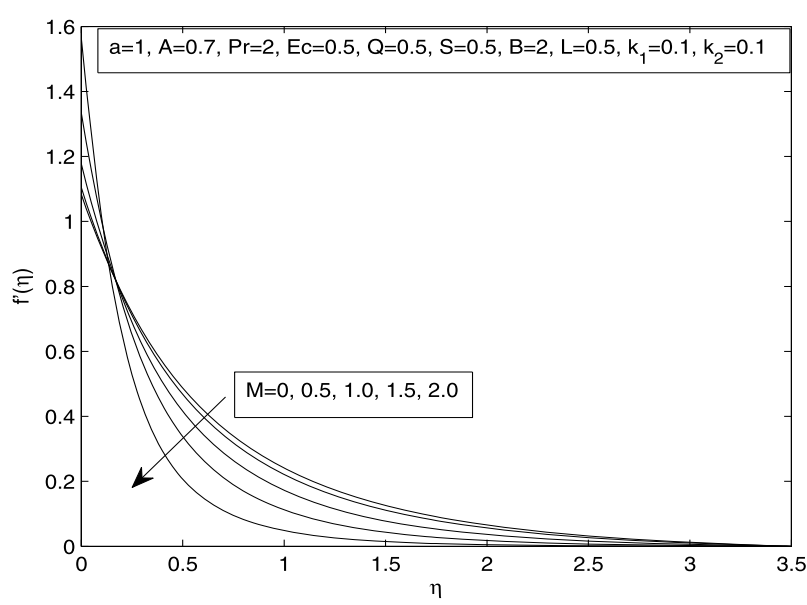

Figure 16 Variation of velocity $f^{\prime}(\eta)$ for different values of Hartmann number $M$.

shows that the increasing Prandtl number reflects a higher capability of heat transfer. The effects of the source $(Q>0)$ and the sink $(Q<0)$ on the temperature $\theta(\eta)$ are shown in Figure 10. It is noticed that the temperature increases if there exists a heat source, and a decrease in temperature is noticed in the case of a sink.

In addition, the effects for the second type of boundary condition are discussed in the following section. For the verification of accuracy of the applied numerical scheme, a comparison of the present results corresponding to $-f^{\prime \prime}(0)$ with the ones obtained by Dessie and Kishan [27], Cortell [29], and Mukhopadhyay et al. [30] is presented in Table 1, which shows a favorable agreement. Table 2 presents the numerical values of skin friction coefficient $-f^{\prime \prime}(0)$ for various values of $M, S$, and $k_{1}$.

Figures 11 and 12 show that the velocity at the sheet is different since there exists slip velocity. In addition, it is a decreasing function of the viscosity parameter and the temperature is an increasing function of viscosity parameter. The effects of slip coefficients on the velocity are shown in Figures 13 and 14. It is observed that the velocity decreases with the first slip coefficient $k_{1}$ but increases with the second slip coefficient $k_{2}$. The effects of the 


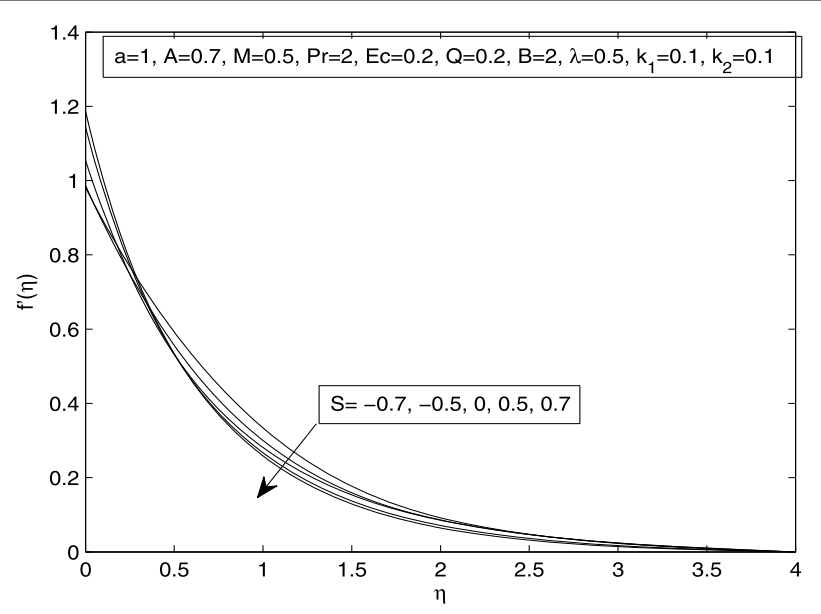

Figure 17 Variation of velocity $f^{\prime}(\eta)$ for different values of suction/injection parameter $S$.

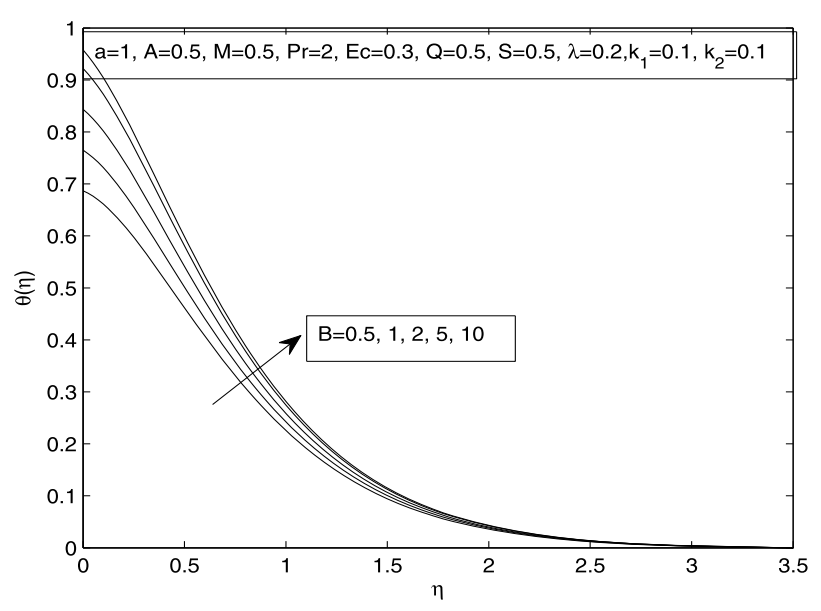

Figure 18 Variation of temperature $\theta(\eta)$ for different values of convection heat transfer parameter $B$.

permeability parameter, the Hartmann number, the suction parameter, the Prandtl number and the Eckert number, and the heat sink/source on the velocity and the temperature distribution are similar to the case corresponding to the first type of boundary conditions. However, there also exists a significant difference, which is the velocity at the sheet. Here the velocity at the sheet is different because of the existence of the slip velocity. All of these are shown in Figures 15-21.

\section{Conclusion}

In this paper, we have considered the similarity solutions of MHD boundary layer flow and convective heat transfer of a fluid with variable viscosity through a porous medium towards a stretching sheet. By determining the transformation group under which the given partial differential equation and its boundary conditions are invariant, we obtain the invariants and the symmetries of this equation. In turn, with the help of these invariants and symmetries, we determine the similarity variables that reduce the number of independent variables. With the two different boundary conditions, the scaling group 


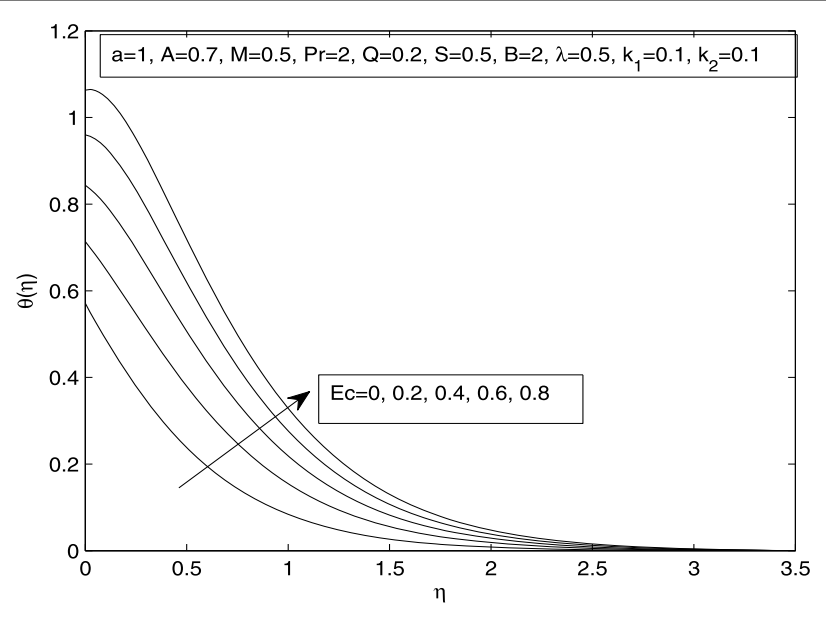

Figure 19 Variation of temperature $\theta(\eta)$ for different values of Eckert number $E c$.

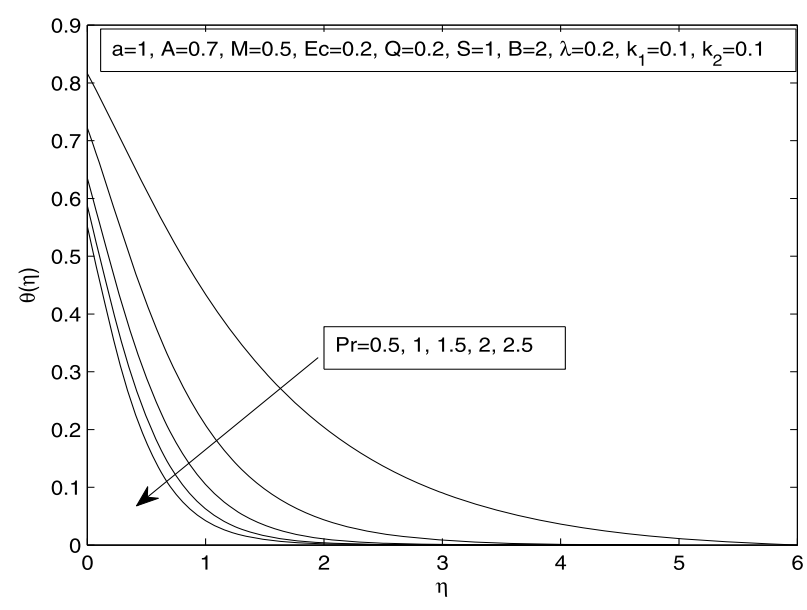

Figure 20 Variation of temperature $\theta(\eta)$ for different values of Prandtl number Pr.

of transformations transformed the governing partial differential equations into two different kinds of boundary value problems, which are solved numerically using MATLAB package and the results are plotted. The influence of different physical parameter on the velocity or temperature distribution are analyzed. The main findings can be summarized as follows:

- The two types of boundary conditions satisfying the similarity solution can be obtained with the assistance of scaling group of transformations.

- As there exists a slip parameter, the influence of various physical parameters, such as the viscosity parameter, the Eckert number, the Prandtl number, the permeability parameter, and the Hartmann number on the velocity and temperature distribution is significantly different from the case of no slip velocity.

- Because of the existence of the slip parameter, the velocity at the plate have different values influenced by Hartmann number, permeability parameter, and viscosity parameter. 


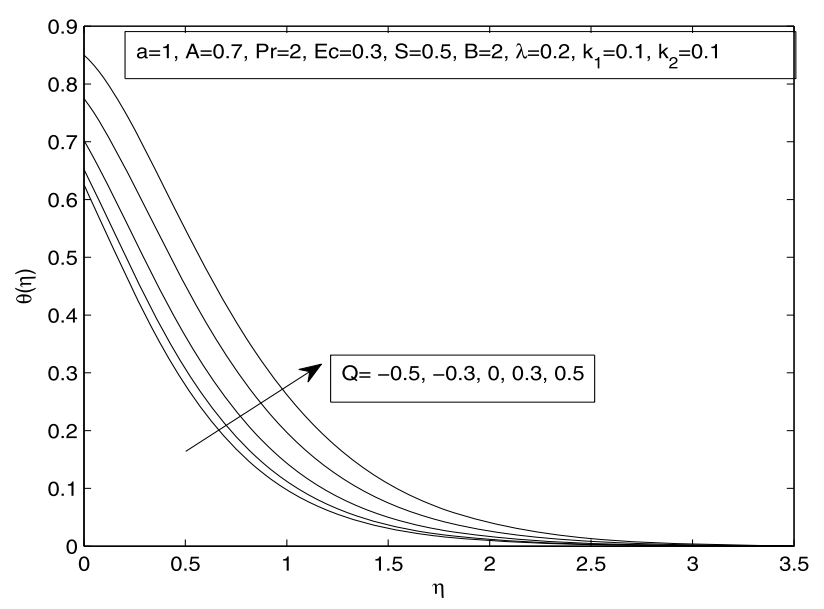

Figure 21 Variation of temperature $\theta(\eta)$ for different values of heat source/sink parameter $Q$.

- Whether there exists slip parameter or not, the temperature distribution is an increasing function of the Eckert number, the convection heat transfer parameter, and the heat source/sink parameter, respectively. However, it is the decreasing function of increasing Prandtl number.

\section{Competing interests}

The authors declare that they have no competing interests.

\section{Authors' contributions}

The authors declare that the study was realized in collaboration with the same responsibility. All authors read and approved the final manuscript.

\section{Author details}

'School of Mathematics and Physics, University of Science and Technology Beijing, Beijing, 100083, China. ${ }^{2}$ College of Science, China Agricultural University, Beijing, 100083, China.

\section{Acknowledgements}

This work is supported by the National Natural Science Foundations of China (No. 11302024, No. 61440058), the Fundamental Research Funds for the Central Universities (No. FRF-TP-14-071A2, No. FRF-BR-13-023) and Beijing Higher Education Young Elite Teacher Project (No. YETP0387, No. YETP0322), Research Foundation of Engineering Research Institute of USTB (No. Yj2011-015).

Received: 19 December 2014 Accepted: 7 April 2015 Published online: 22 April 2015

\section{References}

1. Crane, LJ: Flow past a stretching plate. Z. Angew. Math. Phys. 21, 645-647 (1970)

2. Hashizume, H: Numerical and experimental research to solve MHD problem in liquid blanket system. Fusion Eng. Des. 81, 1431-1438 (2006)

3. Aoyagi, M, Ito, S, Hashizume, H, Muroga, T: MHD pressure drop characteristics in a three-surface-multi-layered channel under a strong magnetic field. Fusion Eng. Des. 85, 1181-1184 (2010)

4. Zhang, C, Zheng, L, Zhang, X, Chen, G: MHD flow and radiation heat transfer of nanofluids in porous media with variable surface heat flux and chemical reaction. Appl. Math. Model. 39, 165-181 (2015)

5. Rasmussen, H: Steady viscous flow between two porous disks. Z. Angew. Math. Phys. 21, 187-195 (1970)

6. Hayat, T, Abbas, Z, Sajid, M: Series solution for the upperconvected Maxwell fluid over a porous stretching plate. Phys. Lett. A 358, 396-403 (2006)

7. Noor, NFM: Analysis for MHD flow of a Maxwell fluid past a vertical stretching sheet in the presence of thermophoresis and chemical reaction. World Acad. Sci., Eng. Technol. 64, 1020-1023 (2012)

8. Hayat, T, Fetecau, C, Sajid, M: On MHD transient flow of a Maxwell fluid in a porous medium and rotating frame. Phys. Lett. A 372, 1639-1644 (2008)

9. Ibrahim, W, Shankar, B: MHD boundary layer flow and heat transfer of a nanofluid past a permeable stretching sheet with velocity, thermal and solutal slip boundary conditions. Comput. Fluids 75, 1-10 (2013)

10. Ovsiannikov, LV: Group Analysis of Differential Equations. Academic Press, New York (1982)

11. Bluman, GW, Kumei, S: Symmetries and Differential Equations. Springer, New York (1989)

12. Olver, J: Application of Lie Groups to Differential Equations. Springer, New York (1989) 
13. Abd-el-Malek, MB, Badrana, NA, Hassanb, HS: Using group theoretic method to solve multi-dimensional diffusion equation. J. Comput. Appl. Math. 147, 385-395 (2002)

14. Boutros, YZ, Abd-el-Malek, MB, Badran, NA, Hassan, HS: Lie-group method for unsteady flows in a semi-infinite expanding or contracting pipe with injection or suction through a porous wall. J. Comput. Appl. Math. 197, 465-494 (2006)

15. Boutros, YZ, Abd-el-Malek, MB, Badran, NA, Hassan, HS: Lie-group method solution for two-dimensional viscous flow between slowly expanding or contracting walls with weak permeability. Appl. Math. Model. 31, 1092-1108 (2007)

16. Abd-el-Malek, MB, Aminb, AM: Lie group analysis of nonlinear inviscid flows with a free surface under gravity. J. Comput. Appl. Math. 258, 17-29 (2014)

17. Mekheimer, KS, El-Sabbagh, MF, Abo-Elkhair, RE: Lie group analysis and similarity solutions for hydro-magnetic Maxwell fluid through a porous medium. Bound. Value Probl. 2012, Article ID 15 (2012)

18. Pakdemirli, M, Yurusoy, M: Similarity transformations for partial differential equations. SIAM Rev. 40, 96-101 (1998)

19. Yurusoy, M, Pakdemirli, M, Noyan, OF: Lie group analysis of creeping flow of a second grade fluid. Int. J. Non-Linear Mech. 36, 955-960 (2001)

20. Mukhopadhyay, S, Layek, GC, Samad, SA: Study of MHD boundary layer flow over a heated stretching sheet with variable viscosity. Int. J. Heat Mass Transf. 48, 4460-4466 (2005)

21. Kanadasamy, R, Muhaimin, I: Lie group analysis for the effect of temperature dependent fluid viscosity and thermophoresis particle deposition on free convective heat and mass transfer under variable stream conditions. Appl. Math. Mech. 31, 317-328 (2010)

22. Zhu, J, Zheng, L, Zhang, Z: Effects of slip condition on MHD stagnation-point flow over a power-law stretching sheet. Appl. Math. Mech. 31, 439-448 (2010)

23. Hamad, MAA: Analytical solution of natural convection flow of a nanofluid over a linearly stretching sheet in the presence of magnetic field. Int. Commun. Heat Mass Transf. 38, 487-492 (2011)

24. Das, K: Lie group analysis for nanofluid flow past a convectively heated stretching surface. Appl. Math. Comput. 221, 547-557 (2013)

25. Kundu, PK, Das, K, Jana, S: Nanofluid flow towards a convectively heated stretching surface with heat source/sink: a lie group analysis. Afr. Math. 25, 363-377 (2014)

26. Asghar, S, Jalil, M, Hussan, M, Turkyilmazoglu, M: Lie group analysis of flow and heat transfer over a stretching rotating disk. Int. J. Heat Mass Transf. 69, 140-146 (2014)

27. Dessie, H, Kishan, N: MHD effects on heat transfer over stretching sheet embedded in porous medium with variable viscosity. viscous dissipation and heat source/sink. Ain Shams Eng. J. 5, 967-977 (2014)

28. Batchelor, GK: An Introduction to Fluid Dynamics. Cambridge University Press, London (1987)

29. Cortell, R: Flow and heat transfer of a fluid through a porous medium over a stretching surface with internal heat generation/ absorption and suction/blowing. Fluid Dyn. Res. 37, 231-245 (2005)

30. Mukhopadhyay, S, Layek, GC, Samad, SA: Effects of variable fluid viscosity on flow past a heated stretching sheet embedded in porous medium in presence of heat source/sink. Meccanica 47, 863-876 (2011)

\section{Submit your manuscript to a SpringerOpen ${ }^{\odot}$ journal and benefit from:}

- Convenient online submission

Rigorous peer review

- Immediate publication on acceptance

- Open access: articles freely available online

- High visibility within the field

- Retaining the copyright to your article 